\title{
Route to Larger Pulse Energy in Ultrafast Fiber Lasers
}

\author{
Luming Zhao, Daojing Li, Lei Li, Xuan Wang, Ying Ge, Deyuan Shen, and Lei Su
}

\begin{abstract}
Principles of pulse shaping aiming larger pulse energy in fiber lasers are reviewed. From conventional solitons generated in anomalous dispersion regime, stretched pulses in dispersion-managed fiber lasers, similaritons in normal dispersion regime, dissipative solitons in large normal dispersion regime, amplifier similaritons in fiber lasers with narrow bandwidth filtering, to dissipative-soliton-resonance (DSR) pulses in fiber lasers, scaling of pulse energy from pico-Joule level to micro-Joule is successfully achieved. DSR is a possible way to achieve endless pulse energy, which is limited by the available pump power and material damage threshold. Properties of DSR pulses are reviewed. Specifically, we experimentally and numerically demonstrate tilt-top DSR pulses in a normal dispersion mode-locked fiber laser. Two fronts of the tilt-top pulses exhibit different intensity, and the intensity disparity maintains while the pulses extend with increasing gain. The tilt-top DSR pulses have rectangle spectral pedestal and a peak. The spectral peak deviates from the center of the rectangle spectral pedestal. Further results show that the asymmetry of two fronts is closely related to the wavelength deviation of spectral peak. Numerical simulations reveal the importance of the saddle-type spectral filtering on asymmetric DSR pulse generation.
\end{abstract}

Index Terms-Fiber lasers, Fiber nonlinear optics, Optical fiber dispersion, Optical pulse generation, Optical solitons

\section{INTRODUCTION}

Ultrafast fiber lasers are the most active research focus among fiber optics within the past forty-five years [1-5]. Benefited from the mature of fiber technology, ultrafast fiber lasers have been extensively exploited in optical communications, optical sensing, laser surgery, material machining, and nonlinear optics. Comparing with traditional solid-state lasers, fiber lasers have intrinsic advantages such as good beam quality, compact structure, reliable operation, low cost maintaining, good heat dissipation etc. Fiber lasers those can output pico-/femto-second pulses have attracted more and more attention in past decade.

The theoretical work on pulse shaping could be dated back to Hasegawa's work in 1973, in which solitons was first predicted to be able to generate in optical fibers [1]. In the presence of anomalous group velocity dispersion, the nonlinear dependence of the index of refraction on intensity can make possible the transmission of picosecond pulses without distortion in fibers. The first experimental

L. Zhao, L. Li, X. Wang and Y. Ge are with the Jiangsu Key Laboratory of Advanced Laser Materials and Devices, Jiangsu Collaborative Innovation Center of Advanced Laser Technology and Emerging Industry, School of Physics and Electronic Engineering, Jiangsu Normal University, Xuzhou, Jiangsu 221116 China, L. Zhao is also with the Key Laboratory of Optoelectronic Devices and Systems of Ministry of Education and Guangdong Province, Shenzhen University, 518060, China (e-mail: zhaoluming@jsnu.edu.cn; leelei@jsnu.edu.cn; tangdingyuan@jsnu.edu.cn).

D. Li and D. Shen are with the Department of Optical Science and Engineering, Fudan University, Shanghai 200433, China (e-mail: daojingli12 @ fudan.edu.cn; shendy@fudan.edu.cn).

L. Su is with the School of Engineering and Materials Science, Queen Mary University of London, London, UK (e-mail: 1.su@qmul.ac.uk). demonstration of soliton generation in fibers are done by Mollenauer et al [2]. A Fourier transform-limited optical pulse with a 7 ps pulse width was used in the input and the output pulse shapes were measured by means of autocorrelation. At critical power level of 1.2 $\mathrm{W}$, the soliton features are observed.

Fiber lasers are constructed mainly by fibers. It is straightforward to expect to extend the knowledges learned from fibers to fiber lasers. Pulse propagation in fibers is described by the nonlinear Schrodinger equation (NLSE) while the pulse propagation in fiber lasers is generally expressed by the Ginzburg-Landau equation. By taking certain hypothesis, for example, the gain-loss effect can be considered as a weak perturbation to the system, it is feasible to use the NLSE to describe pulse dynamics in a fiber laser. Several preliminary work on soliton generation in fiber lasers were reported a decade later than soliton generation in fibers [3-5]. By operating the cavity dispersion in the anomalous regime, stable ultrashort pulses or solitons can be generated in fiber lasers. Pulse duration of 4 ps [3], $430 \mathrm{fs}$ [4], and $320 \mathrm{fs}$ [5] are respectively achieved.

It is now well known that solitons generated in fiber lasers are due to the balanced interaction between the anomalous dispersion effect and the nonlinear Kerr effect imposing on pulse during propagation. Fibers have small core diameter, which causes strong power flux. Together with long interaction length between light and fiber segments, the accumulation of nonlinear phase shift will be significant especially when the pulse peak power of ultrashort pulses increases. Excess nonlinear phase shift accumulation will result in spectrum broadening, consequently pulse deformation and wave breaking will be induced, which limits the single pulse energy achievable. It is the key factor that seriously affects the performance improving of fiber lasers. Larger pulse energy means higher peak power for a same pulse duration. To overcome this limitation and to achieve larger pulse energy, various pulse shaping mechanisms are proposed and demonstrated [***]. In this paper, we summarize the different pulse shaping mechanisms aiming larger pulse energy. In section II we explain the stretched-pulse or dispersion-managed soliton generation in fiber lasers. In section III we describe the principle of similariton generation in fiber lasers. In section IV we introduce the concept of dissipative solitons in large normal dispersion regime. Amplifier similaritons in fiber lasers with narrow bandwidth filtering are briefly discussed. In section $\mathrm{V}$ we elaborate dissipative-soliton-resonance (DSR) pulse generation in fiber lasers and their properties. In section VI we show both experimental and numerical demonstration of tilt-top DSR pulses in a normal dispersion mode-locked fiber laser. The temporal and spectral characteristics of tilt-top DSR pulses will be explored: the two fronts of the tilt-top pulses exhibit different intensity, and the intensity disparity maintains while the pulses extend with increasing gain. In frequency domain, the spectral peak deviates from the center of the rectangle spectral pedestal. In addition, the asymmetry of two fronts in pulse profile is closely related to the wavelength bias of spectral peak. A saddle-type spectral filtering is required for the asymmetric DSR pulse generation. Section VII summarizes our main conclusion. 


\section{Dispersion-Management In Fiber LASERS}

Due to the area theorem limitation $[1,6,7]$, which expresses the pulse energy as a function of the pulse duration and the laser parameters, traditional solitons could only have pulse energy up to tens of pico-Joule. $11 \mathrm{pJ}$ output pulse energy and $22 \mathrm{pJ}$ intracavity pulse energy was obtained in [3], $16 \mathrm{pJ}$ external pulse energy and 52 pJ intracavity pulse energy was achieved in the all-fiber laser [5]. Increasing pump power increases the soliton number instead of pulse energy increase. To obtain larger pulse energy, dispersion management is first adopted by employing pulse compression mode-locking with help of gratings [4]. Output pulse energy of 172 $\mathrm{pJ}$ and $850 \mathrm{pJ}$ intra-cavity pulse energy was achieved. Tamura et al adopted the dispersion-managed design and realized it in an all-fiber laser [8]. A fully self-starting all-fiber laser outputting 77 fs pulse with $90 \mathrm{pJ}$ was successfully demonstrated. The intracavity pulse energy at the output position is about $900 \mathrm{pJ}$.

The physical mechanism of stretched-pulse or dispersion-managed soliton generation is as the following. As shown in Fig. 1, instead of constructing the entire fiber laser from negative dispersion fibers only [6], fibers with different sign of dispersion are used. Here the erbium-doped fiber (EDF) has positive dispersion, while all the other fibers have standard negative dispersion at wavelength around 1550 $\mathrm{nm}$. During propagation in the positive dispersion fiber, pulses experience large amounts of spreading. The pulse can breathe by an order of magnitude of pulse duration within the cavity (where the term of "stretched pulse" comes from), which lowers the average peak power compared with that which would be experienced by an unchanging transform-limited pulse of the same bandwidth. Therefore the nonlinear phase shift accumulation is effectively reduced. Consequently, 1 order of pulse energy improvement could be achieved. By taking the dispersion management design, a stretched-pulse laser stabilized by a nonlinear optical loop mirror (NOLM) that produces $1-n J$ and $100-f$ pulses was demonstrated [11]. However, further increasing of the pump energy still results in multiple pulsing.

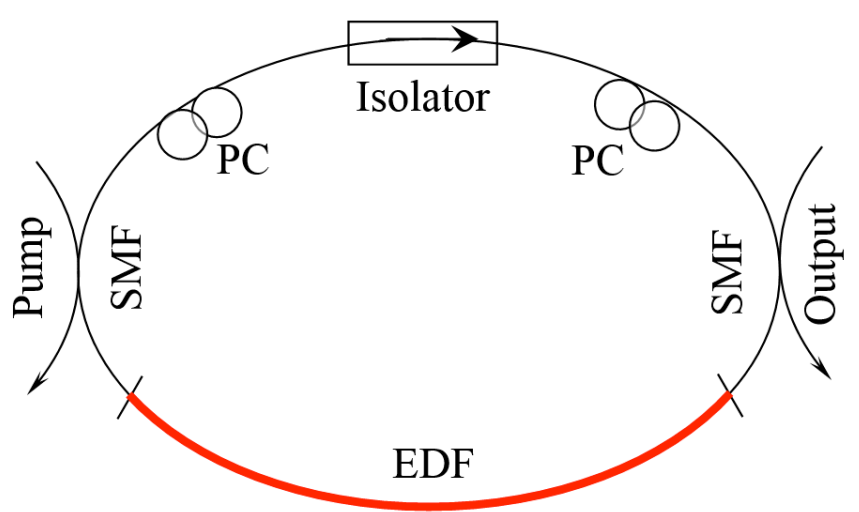

Fig. 1 Schematic of dispersion management. PC: polarization controller; SMF: single-mode fiber; EDF: erbium-doped fiber [10].

Similar to the concept of dispersion management, Ilday et al proposed to introduce nonlinearity management in fiber lasers by the use of self-defocusing nonlinearities to control nonlinear phase shift in soliton fiber lasers [12]. It is possible to increase the pulse energy by 2 orders of magnitude. However, it is difficult to find suitable materials with required nonlinearity.

\section{Similariton Generation In Fiber LASERS}

To avoid multiple pulsing generation in fiber lasers and further improve single pulse energy, scientists attempted different ways. One of them is to look for an analytical solution of the NLSE, which can maintain wave-breaking-free under scaling rules. Self-similarity is a term to describe recurring phenomenon. Self-similar propagation of short pulses of parabolic intensity profile was first demonstrated in optical fibers with normal group-velocity dispersion and strong nonlinearity [13]. Pulses with a parabolic intensity variation and a linear frequency chirp can be an approximate wave-breaking-free solution of the NLSE in the high intensity limit. Fermann and Harvey et al extended the concept to an optical fiber amplifier with normal dispersion [14-17]. It is found that there exists an exact asymptotic solution corresponding to a linearly chirped parabolic pulse which can propagate self-similarly. The amplified pulse can maintain parabolic profile when further propagating in standard single mode fiber with normal dispersion.

The above-mentioned work makes a solid foundation and a straightforward direction to achieve high power parabolic pulses in a fiber laser with normal dispersion. By carefully designing a fiber laser, Ilday et al theoretically and experimentally demonstrate self-similar evolution of parabolic pulses in most of the fiber laser [18]. The periodic boundary condition of the laser is satisfied by using a dispersive delay line with anomalous dispersion and negligible nonlinearity. Parabolic pulse with $10 \mathrm{~nJ}$ was obtained. The cavity is dispersion-managed. However, the pulse evolution in cavity is typically different from that of dispersion-managed solitons. Breathing happens only once instead of twice for dispersion-managed solitons [19]. By exploiting the self-similar operation in an Yb-fiber laser, pulse energy up to $14 \mathrm{~nJ}$ was achieved [20]. The pulses can be dechirped to sub-100-fs duration with peak power of $100 \mathrm{~kW}$.

Self-similar propagation of intense pulses will be disrupted if the pulse encounters any limitation to its spectral bandwidth [17]. Due to the monotonic evolution of self-similar pulses and cavity boundary condition in a laser, any changes making to the pulse after traversal of the cavity should be reversed to restore the self-similar solution. Numerically it is found that, under appropriate cavity parameter settings, it is able to achieve pulse breaking recovery in fiber lasers, where the local pulse breaking in the cavity could be recovered during pulse propagation in the gain fiber with normal dispersion [10]. Transform-limited pulses with bandwidth beyond the laser gain bandwidth was numerically demonstrated in the laser.

\section{Dissipative Soliton Generation In Fiber Lasers}

Traditional soliton theory considers only the anomalous dispersion regime as a bright pulse propagating without distortion can be obtained with the help of fiber nonlinear effect [1]. Normal dispersion regime can support dark soliton generation only within the frame of NLSE [21]. Solitons are generated due to balanced interaction, which triggers us to consider the possibility to achieve soliton behaviors in fiber lasers with normal dispersion. In the normal dispersion regime, the dispersion effect and the fiber nonlinear effect play a same role on pulse, it is still possible to obtain undistorted pulses if there exists a mechanism to appropriately compensate the combined influence from the normal dispersion effect and the nonlinear effect [22].

Spectral filtering with appropriate bandwidth is the first attempt to be considered as the normal dispersion effect and the nonlinear Kerr effect imposing on pulse play the same role to broaden the pulse in frequency domain [23]. An EDF laser made of fibers with pure normal dispersion was first tested [24]. Stable chirp pulses with characteristically steep spectral edges are obtained as shown in Fig. 2. Pulse energy up to $0.55 \mathrm{~nJ}$ (5.5 nJ intracavity) was obtained with maximum available pump power of $\sim 700 \mathrm{~mW}$. Spectral filtering is 
provided by the gain bandwidth limitation or gain dispersion effect as the gain bandwidth of an EDF is about $20 \mathrm{~nm}$. Therefore, the pulses were called "gain-guided solitons (GGSs)." After the successful demonstration of stable pulses generated in EDF lasers of normal dispersion, Chong et al achieved similar pulse performance in Yb-fiber lasers where the spectral filtering is provided by an interference filter with $10 \mathrm{~nm}$ bandwidth [25]. Apart from the dissipative process of gain-loss balance, the dissipative process of spectral filtering play a crucial role for stable pulse generation in the normal dispersion fiber laser. Therefore, the stable pulses are also called "dissipative solitons (DSs)." Renninger et al. found that there exists an exact particular solution of the cubic-quintic GLE models pulses in the normal-dispersion laser [26].
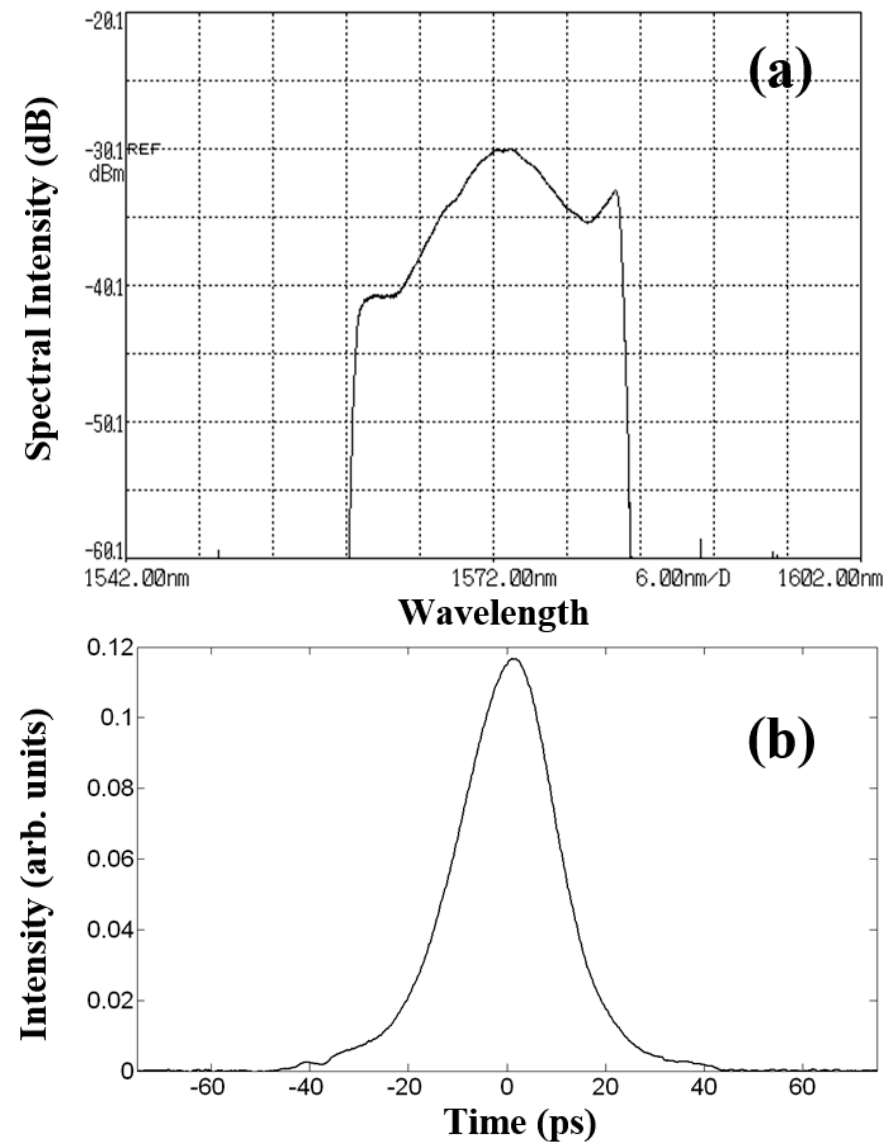

Fig. 2 (a) Typical optical spectrum and (b) corresponding autocorrelation trace of the gain-guided soliton [24]

A series of research on the properties of GGSs [27-39] and DSs are carried out [40-46]. It is found that, similar to the traditional soliton generated in fiber lasers with anomalous dispersion, GGSs can express various soliton features. GGSs can be generated in dispersion-managed fiber lasers provided the net cavity dispersion is large normal [27]. By combining the nonlinear compression with nonlinear amplification, 47 fs pulse was directly generated from an EDF laser [28, 29]. The state of GGSs and that of noise-like pulses can be shuttled with appropriate operation condition tuning [30]. Based on GGSs, phenomena of multiple pulsing [31], bound states [32], harmonic mode locking [33], period-doubling [34], soliton trapping [35] could be observed. The dynamics of GGS generation under various conditions are also explored [36-39]. It is numerically demonstrated that broad gain bandwidth up to $100 \mathrm{~nm}$ can still support DS generation subject to enough pump power [40].
Kieu et al. exploited DS formation with cladding pumping and demonstrated the first fiber laser to reach the performance of standard commercially available Ti:sapphire lasers [42]. The laser could generate $31 \mathrm{~nJ}$ chirped pulses. After dechirping outside the laser, 80 fs pulses, with $200 \mathrm{~kW}$ peak power, are obtained. By exploiting a large-mode-area $\mathrm{Yb}$-doped photonic crystal fiber, Lefrancois et al achieved a DS with pulse energy of $140 \mathrm{~nJ}$ and the pulse could be dechirped to 115 fs [43]. Baumgartl et al used a same type of fiber but with different parameter in a sigma cavity, the watt-level laser directly emits DSs with a duration of $1 \mathrm{ps}$ and $163 \mathrm{~nJ}$ of pulse energy [44]. The larger pulse energy is obtained with the sacrifice of all-fiber design.

DS generation in fiber lasers is described by the GLE considering dissipative processes especially the spectral filtering. Renninger et al found that, when a spectral filter of narrow bandwidth is used in an all-normal-dispersion fiber laser, self-similar pulse evolution can be achieved, which is a new regime of mode locking [48]. The restoration of the self-similar evolution is completed by the narrow spectral filtering before the gain bandwidth significantly affects the self-similar evolution.
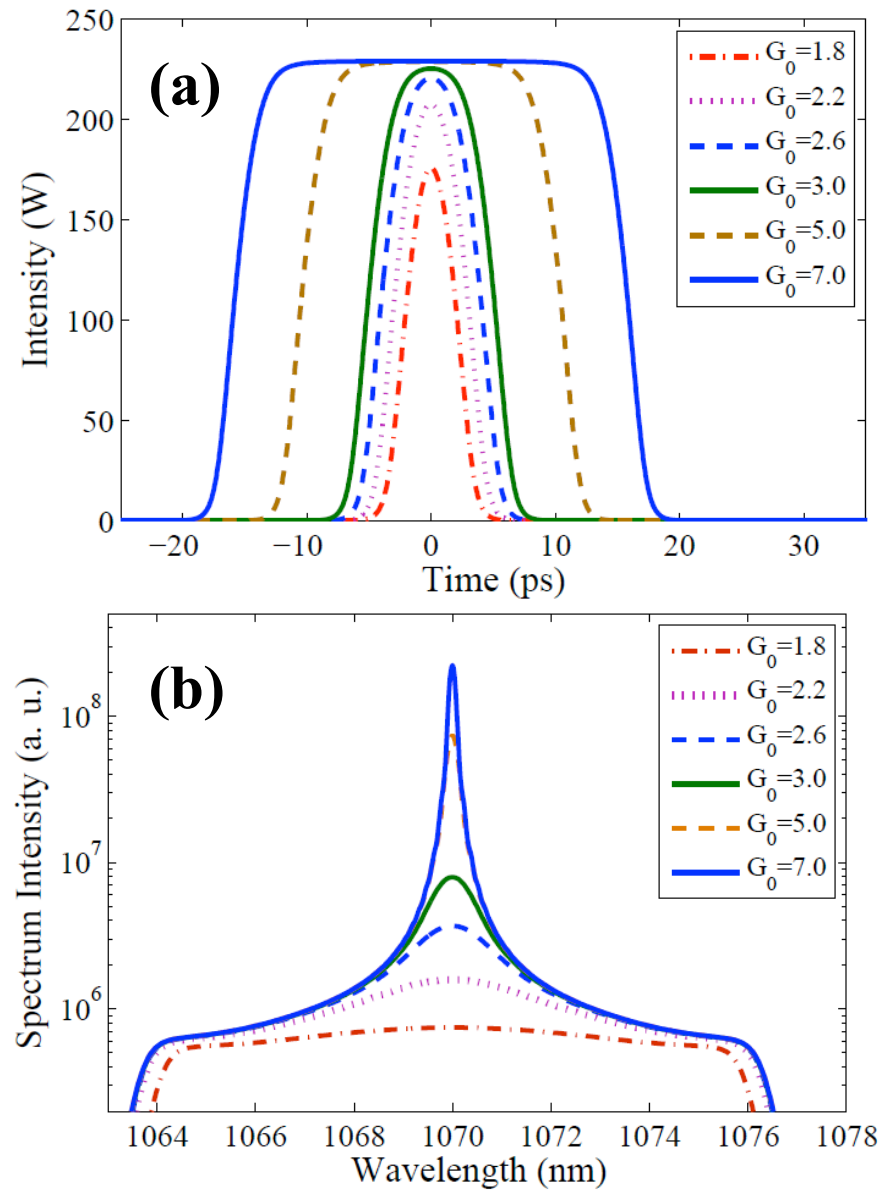

Fig. 3 Typical DSR pulse profile (a) and spectrum (b) variation versus increasing pump [50]

\section{Dissipative-Soliton-Resonance In Fiber LASERS}

The successful demonstration of pulse energy boost in the normal dispersion regime attracts a lot of attention. However, the further pulse energy increase is still stopped by the multiple pulsing generation resulted from the excess of nonlinear phase shift accumulated. In 2008, Chang et al. [49] proposed a new concept of 
soliton formation, the so-called "dissipative soliton resonance" (DSR), in the frame of complex cubic-quantic GLE with certain parameter settings. Within this parameter regime, the pulse peak power remains constant. It means arbitrarily pulse energy increase is possible with broadened pulse width. The nonlinear phase shift could be dramatically reduced with low pulse peak power. Further theoretical work suggests that the DSR operation can also happen in the anomalous dispersion regime [50]. Fig. 3 shows a typical DSR pulse profile and spectrum variation with increasing pump level [51].

Ding et al. proved that the DSR was achievable with realistic laser settings [52]. Komarov et al. investigated the competition and coexistence of ultrashort laser pulses under the DSR conditions [53]. They found that the number of the DSR pulses in steady state operation depends on the initial conditions, but would not change with increasing pump power. The DSR generation has also been experimentally observed in fiber lasers mode-locked by the nonlinear polarization rotation technique [54] and the NOLM [55-57].

$\mathrm{Li}$ et al proposed a theoretical model to numerically study the mechanism of the multipulse operation and the DSR generation in a fiber laser [51]. Pulses propagating in the fiber experience nonlinear spectral broadening. Higher peak power leads to broader spectral width. For lasers with monotonic saturable absorption, the spectral filtering effect, which limits the spectral maximum width, causes the multipulse operation in the dissipative soliton laser. Laser cavities with larger spectral filter bandwidth favor pulses with broader spectrum and higher peak power. To achieve the DSR generation in the cavity, strong peak-power-clamping effect of a sinusoidal saturable absorption or a low-threshold anti-saturable absorption is required. When the cavity peak-power-clamping effect is strong enough that the pulse peak power and the pulse spectral width are both confined at a low value, the DSR is generated taking precedence of multipulse generation. Otherwise, the spectral filtering effect causes pulse breaking before the pulse peak power reaches the saturation point.

The DSR pulses have rectangular spectral base with characteristic steep edges as shown in Fig. 3(b). Detailed studies [58] suggest that the rectangular part of the spectrum is almost unchanged while the newly generated spectral component locates at the center and forms a peak with increasing energy. It is found that a DSR pulse has two different chirps along its profile: a very low linear chirp throughout the extended pulse plateau and large linear chirps across both edges of the pulse. During compression, the DSR pulse firstly evolves into a cat-ear profile with a unique crown shape autocorrelation curve before being compressed to the narrowest width. DSR pulse could be compressed down to femtosecond pulse duration. However, special compensating dispersion design should be considered.

Pulse energy of $\sim 70 \mathrm{~nJ}$ was obtained for DSR pulses from a linear cavity fiber laser [59]. Recently, the energy of a single DSR pulse, directly from a laser cavity, has been boosted up to $10 \mu \mathrm{J}$ by using a double-clad fiber amplifier to form a nonlinear amplifying loop mirror [60]. Due to the low peak power, DSR pulses are perfect candidate for amplification. Amplified DSR pulses of tens of micro-Joules have been demonstrated [61]. DSR pulses can also be achieved in an all-polarization-maintaining fiber laser [62].

\section{TILT-Top DSR Pulses ANd Their Properties}

DSR operation in fiber lasers paves a way for achieving unlimited pulse energy. Therefore, it is desired to know the detailed dynamics of DSR operation under various conditions and the properties of DSR pulses is required to be understood thoroughly.

DSR can happen in either normal dispersion region [49] or anomalous dispersion regime [50], however, exhibiting different characteristics. The anomalous dispersion provides natural balance of convention solitons. Large efforts have been devoted into DSR in normal dispersion region whereas more complex balance of energy exchange is required and therefore more exotic nonlinear dynamics may be expected. The complex cubic-quintic GLE is widely used in theoretical studies for DSR, unveiling its unique features. Temporally, with peak power fixed, the resonant pulse evolves from Gaussian to flat-top profile. The central plain wave and the fronts were found to feature different chirps: a moderately low linear chirp throughout the extended central plain wave and large linear chirps across both fronts $[58,63]$. Spectrally, a DSR pulse shows a spectrum with broadband rectangular pedestal which corresponds to the fronts. As energy increases, the rectangle part of the spectrum nearly does not change, while the newly emerging spectrum due to the extended central plain wave, appears on the top of the pedestal and forms a narrow peak. DSR pulses with square shape have been demonstrated in a variety of laser cavities. With negligible spectral filtering, the spectral peak should appear at the center of the optical spectrum of the DSR pulse.

In this section, we report on the observation of asymmetric DSR pulses in normal dispersion mode-locked fiber laser. Like regular DSR pulses, spectra of the asymmetric DSR pulses have rectangular pedestal and a peak. However, the asymmetric DSR pulse distinguishes itself by the fact that its peak wavelength does not accord with the center of the rectangular pedestal, therefore the spectrum exhibits asymmetric top. Meanwhile, two fronts of the pulses show different intensity. Consequently, the pulse presents a tilted top instead of a flat top. Further results reveal that if the spectral peak appears at the short (long) wavelength side of the center of rectangular pedestal, the leading front has higher (lower) intensity than the trailing front, and the pulse shows right-angled (left-angled) tilt top. Numerical simulations reveal the importance of the saddle-shape spectral filtering to asymmetric DSR pulses generation.

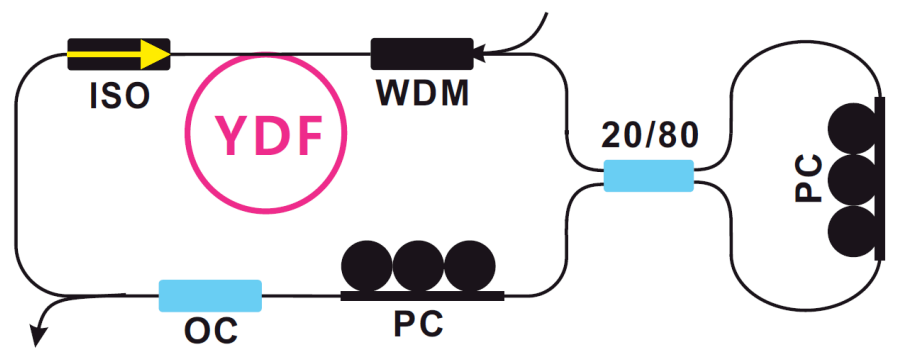

Fig. 4 Schematic of the laser setup. WDM: wavelength division multiplexer; ISO: isolator; PC: polarization controller; YDF: ytterbium-doped fiber; SMF: single-mode fiber; OC: output coupler.

The laser is shown in Fig. 4 with a ring cavity of $17.9 \mathrm{~m}$. The gain was provided by a $42 \mathrm{~cm}$ ytterbium-doped fiber (YDF), counter-pumped by a $976 \mathrm{~nm}$ laser diode. A polarization-independent isolator ensures unidirectional operation. An 80:20 coupler and a 7-meter-long SMF were connected, thus forming a NOLM to provide saturable absorption. The split ratio of the coupler and the length of loop were chosen so to ensure strong peak-power-clamping effect. This is crucial for achieving DSR. Two polarization controllers (PCs) were placed in the cavity and the loop, respectively, providing careful optimization of the cavity birefringence. The laser was output from a $30 \%$ coupler and then monitored by a high-speed photodetector-oscilloscope combination with a bandwidth of $45 \mathrm{GHz}$.

With this laser configuration, flat-top DSR pulses that are predicted in the frame of complex cubic-quintic GLE can be easily observed in the cavity with enough pump power. Yet with different 
PC settings, pulses with tilted top were also observed. Figure 5a summarizes the temporal shapes of one left-tilt pulse with pump power increased from $400 \mathrm{~mA}$ to $700 \mathrm{~mA}$, and Fig. 5b shows the spectrum of the pulse at the pump power of $700 \mathrm{~mA}$. The tilt-top pulse shows essential resemblance to the flat-top DSR pulses, in the following aspects: i) The pulse peak power is limited. With increasing gain, no pulse breaking is observed. Instead, two fronts gradually move away from each other. Pulse width and energy show linear growth with the pump power. ii) Spectrally, the tilt-top pulse also shows rectangular pedestal. As pump power increases, the rectangular pedestal self-maintains while the newly emerging spectrum forms a peak on the top. Those are key features of the DSR pulses. Hence, there are good reasons to consider that the tilt-top pulse still achieves the resonance.
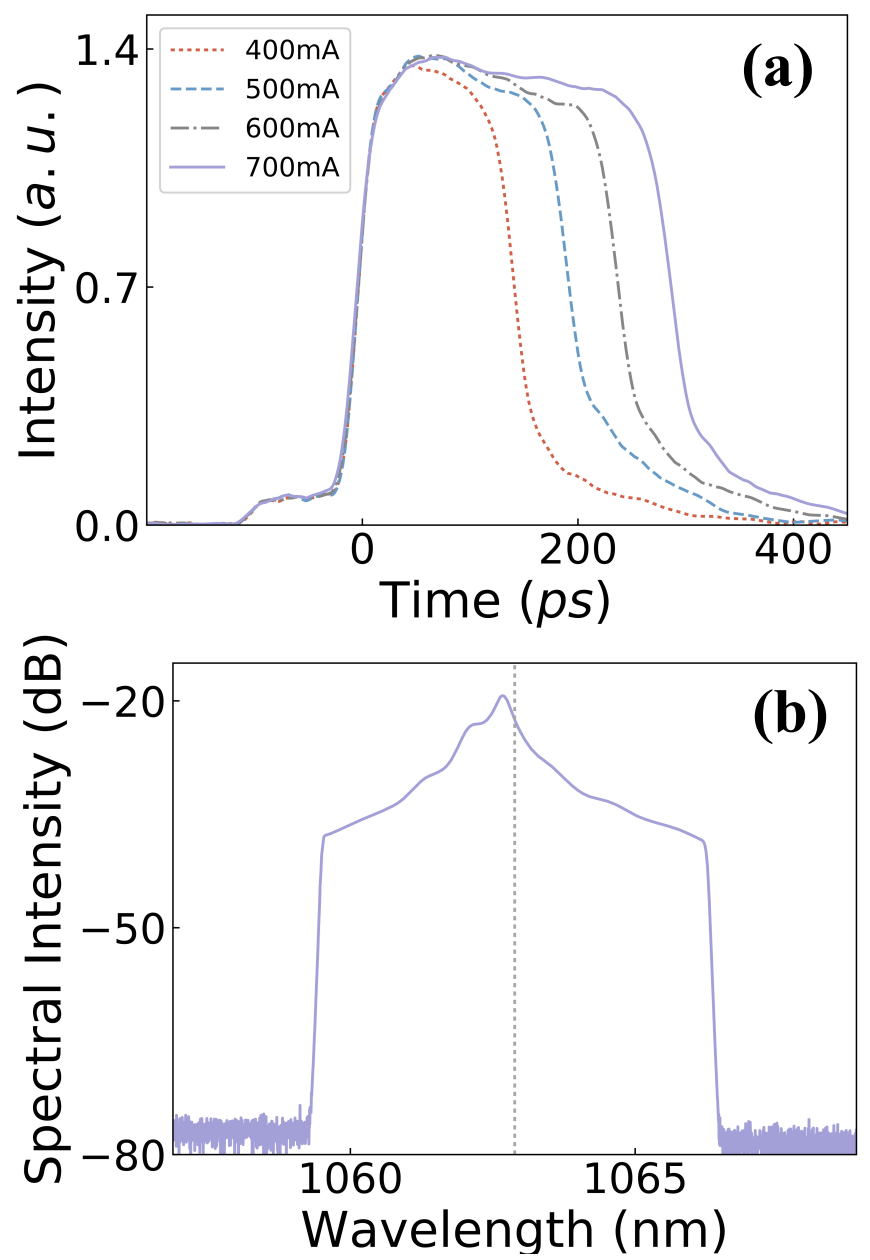

Fig. 5 (a) Temporal profiles of one left-tilt pulse with increasing pump power. (b) Spectrum of the pulse at the pump power of $700 \mathrm{~mA}$. Grey dotted line represents the center of rectangle pedestal.

However, the pulse distinguishes itself from the normal DSR pulses in the complex cubic-quintic GLE by its tilted top. Two fronts of the pulse are no longer symmetric. The leading front has slightly higher intensity than the trailing front. Nonetheless, with increasing gain, two fronts do not show appreciable changes while moving apart from each other. The intensity disparity between two fronts maintains while the whole structure extends. Consequently, the slope of the top of the pulse gradually decreases. The spectrum also shows a major distinction. We recall that for a flat-top DSR pulse in the complex cubic-quintic GLE, its spectral peak lies in the top center of the rectangular pedestal. However, as one can see from Fig. 5b, the peak wavelength of the tilt-top pulse does not accord with the center of the rectangular pedestal (indicated by a grey dotted line). Instead, the peak appears in the short-wavelength side, and the spectrum exhibits an asymmetric top. The short-wavelength part is stronger than the long-wavelength part.
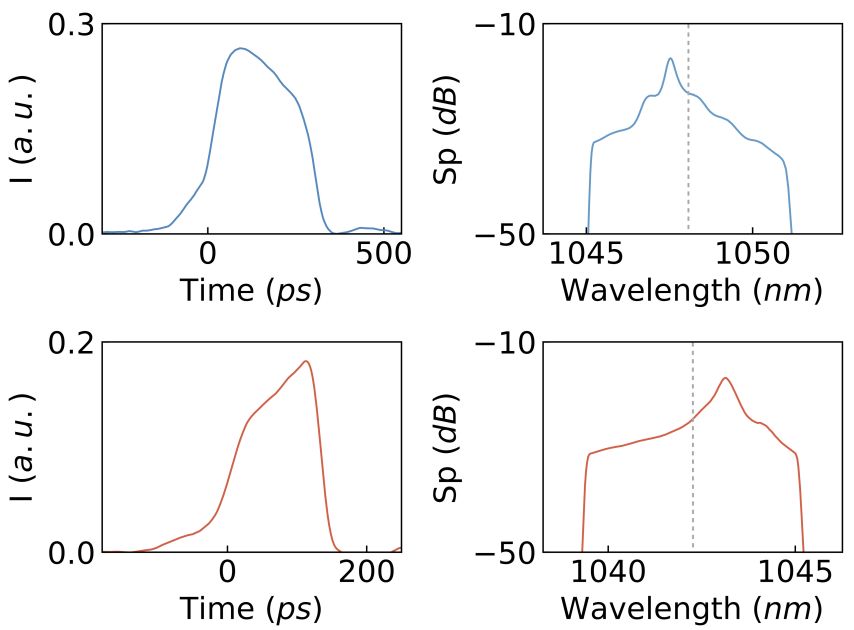

Fig. 6 Two examples of tilt-top pulses with more distorted spectra. Top: left-tilt DSR pulse. Bottom: right-tilt DSR pulse. Left: temporal shape. Right: spectrum, grey dotted line represents the center of rectangular pedestal.

Experimentally, tilt-top DSR pulses always come with distorted spectrum. The asymmetry of two fronts is closely related to the wavelength shift of spectral peak. The top row of Fig. 6 shows another tilt-top DSR pulse, with more distorted spectrum. Comparing to the pulse in Fig. 5, the peak wavelength is shifted more into the short-wavelength region, and two fronts of the pulse exhibit larger intensity difference. Moreover, for pulses whose spectral peaks are shifted to the short wavelength side, the leading fronts always have higher intensity then the trailing fronts, which means the pulses present left-tilt top. When the peaks are shifted to long-wavelength side, the pulses always present right-tilt top. The bottom row of Fig. 6 plots an example of right-tilt pulse. Its spectral peak is shifted to the long-wavelength side and the trailing front has higher intensity.

The experiment results can be considered as clear evidence that spectral filtering of the cavity should play a role on the spectrum of the DSR pulses and meanwhile caused the asymmetry of pulse fronts, which is not seen from the complex cubic-quintic GLE. The complex cubic-quintic GLE has already been proved to be very useful for describing complicated pulse dynamics in mode-locked fiber lasers. However, one restriction of the complex cubic-quintic GLE is that spectral filtering in the model is limited to a perfect parabolic spectral response with a single maximum. In fact, actually spectral response of a mode-locked fiber, like the fiber laser we used here, usually is a combined interaction of various filters in the cavity, such as gain filter, filters of all fiber components, and birefringent filter when the cavity is birefringent. Pulse shapes and spectra are then shaped according to the more complicated spectral filter. In the experiment, when we adjusted the PC settings, the birefringent filter was also changed, so was the overall spectral response of the cavity. Therefore, with different PC sets, pulse shapes and spectra were distorted differently.

To further investigate the influence of cavity spectral response on the resonant pulses, we carry out numerical simulation of the pulse dynamics based on the complex Swift-Hohenberg equation (CSHE), since the addition of a fourth-order spectral filtering term is essential to model more realistic spectral response. The CSHE can be written 
in the following form [64]:

$$
\begin{aligned}
& i \psi_{z}+\frac{D}{2} \psi_{t t}+|\psi|^{2} \psi+v|\psi|^{4} \psi \\
& =i g \psi+i \varepsilon|\psi|^{2} \psi+i \mu|\psi|^{4} \psi+\left(i \delta \psi+i \beta \psi_{t t}+i \sigma \psi t t t t\right)
\end{aligned}
$$

where $\psi$ is the normalized envelope of the field, $\mathrm{t}$ is the retarded time. $D$ is the group velocity dispersion (GVD) coefficient, with its sign dependent on whether the GVD is anomalous or normal. $v$ is the quintic nonlinearity, which in particular can be set as zero. $g$ is the saturable gain including the linear loss $\delta_{0}$ :

$$
g=\frac{g_{0}}{1+\int|\psi|^{2} d t / E_{L}}-\delta_{0}
$$

where $\mathrm{g}_{0}$ is the small-signal gain and $\mathrm{E}_{\mathrm{L}}$ is the saturation energy. $\varepsilon$ and $\mu$ account for the nonlinear loss which arises from saturable absorption. In the case of positive $\varepsilon$ and negative $\mu$, the growth of the pulse intensity is then limited at the value $\varepsilon / \mu$.

The spectral response now is described by the terms involving $\delta$, $\beta, \sigma$ as the spectral transmission $T(\omega)=\exp \left(\delta-\beta \omega^{2}+\sigma \omega^{4}\right)$. When $\beta$ and $\sigma$ are both negative, the spectral response has two maxima at $\omega_{m}= \pm \sqrt{\beta / 2 \sigma}$. For clarity, the spectral filters are illustrated in Fig. 7, where the left plot shows the spectral filter in the CGLE (with $\delta=0$ and $\sigma=0$ ), and the right plot shows the spectral filtering cureves in the CSHE with varying $\omega_{\mathrm{m}}$, modeling the combined spectral response of two interacting spectral filters with different separations. The parameters are chosen in such a way that the heights of two spectral responses are equal and the widths are close.

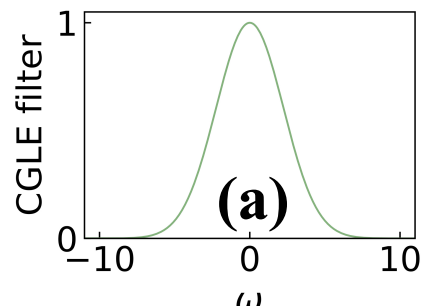

$\omega$

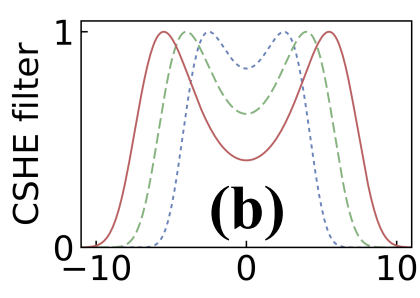

$\omega$
Fig. 7 Spectral filtering $T(\omega)=\exp \left(\delta-\beta \omega^{2}+\sigma \omega^{4}\right)$ in: (a) CGLE with

$\delta=0, \beta=0.1, \sigma=0$; (b) CSHE, $\beta=-0.06$, blue dotted: $\omega_{\mathrm{m}}=2.5 ; \delta=-0.1875$; green dashed: $\omega_{\mathrm{m}}=5 ; \delta=-0.48$; blue dotted: $\omega_{\mathrm{m}}=7.5 ; \delta=-0.9075$.

The equation is solved by split-step Fourier method, using white noise as an initial condition. We first solve the equation using the CGLE filter as plotted in Fig. 7a. We tuned other parameters to $\mathrm{D}=-1.0, \nu=0, \delta_{0}=1.0, \mathrm{E}_{\mathrm{L}}=100, \varepsilon=0.35, \mu=-0.05$ to obtain DSR pulse formation. The result shows flat top and symmetric spectrum, which are common features of the DSR in the CGLE. Fixing other parameters, we then switch to the more complicated CSHE spectral filtering curves with two maxima $\omega_{\mathrm{m}}= \pm 2.5$, as the blue dotted line in Fig. 7b. In this case, the initial white noise can converge to two mirrored states: left-tilt and right-tilt DSR pusles. We summarizes pulse shapes, chirps, spectra of left-tile and right-tile DSR pulses with increasing gain, in the left and right column of Fig. 8, respectively. For the left-tilt DSR pulse, its spectrum is distorted and the peak is shifted to the short-wavelength (high-frequency) side of the center of the spectral pedestal. Note that frequency and wavelength have inverse relationship. The short-wavelength part is stronger than the long-wavelength part, and the leading front has larger intensity than the trailing front. With increasing gain, the pulse extends without breaking while two fronts remain unchanged. And the slope of its top gradually decreases. The frequency chirp also corresponds to these behaviors. The right-tilt DSR pulse is a mirror image of the left-tilt one. Because the spectral response is symmetric around the reference frequency, the initial white noise has equal chance to converge to either one of the two tilt-top pulses. Apart from stable converged states, switching and competition between two cases can also be observed at certain parameter regions. To break this spectral symmetry, we add a very small, e.g., positive (or negative) third-order derivative on the right-hand side of Eq. 1. By doing that, different initial conditions will all converge to the left-tilt (or right-tile) pulse, without significant changes of pulse shape and spectrum.
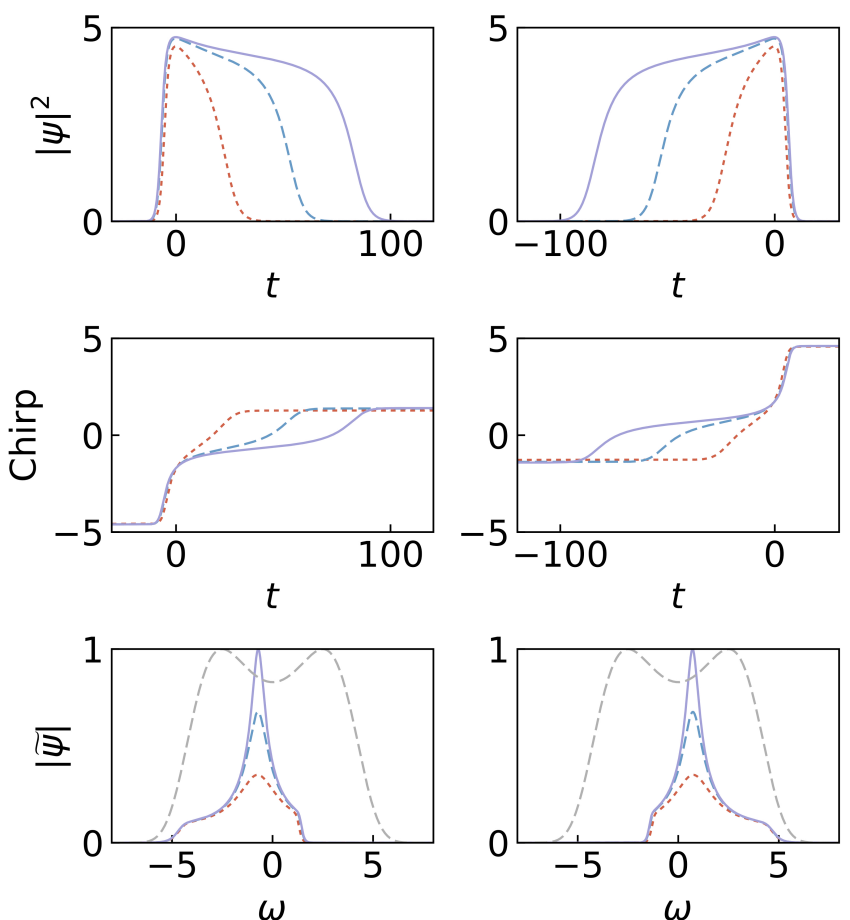

Fig. 8 Numerical simulations of CSHE with increasing gain, $\mathrm{g}_{0}=1.25$ (red dotted lines), $\mathrm{g}_{0}=2$ (blue dashed lines), $\mathrm{g}_{0}=2.75$ (purple solid lines). The spectral filter curve used is indicated by grey dotted line, with two maxima $\omega_{\mathrm{m}}= \pm 2.5$. Left column: left-tilt DSR pulse; Right column: right-tilt DSR pulse; Top: temporal shape; Middle: frequency chirp; Bottom: spectrum 

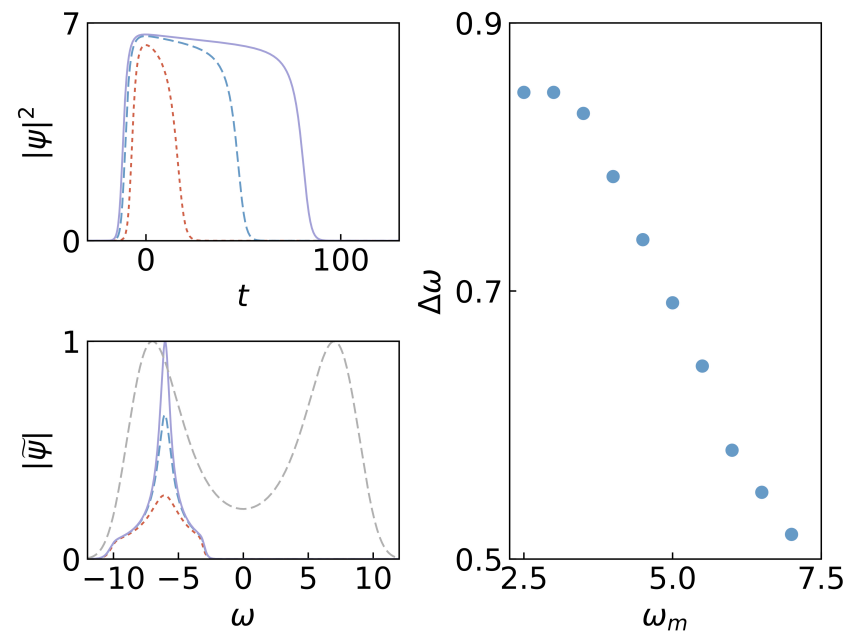

Fig. 9 Left: numerical simulations of CSHE with increasing gain, $g_{a}=2$ (red dotted lines), $\mathrm{g}_{0}=4$ (blue dashed lines), $\mathrm{g}_{0}=6$ (purple solid lines). The spectral filter curve used is indicated by grey dotted line, with two maxima $\omega_{\mathrm{m}}=7.0$. Right: frequency deviation $\Delta \omega$ as a function of $\omega_{\mathrm{m}}$.

We then solve the equation with the CSHE filter with increasing $\omega_{\mathrm{m}}$. The separation of two maxima of the filter carve increases as we increase $\omega_{\mathrm{m}}$, as shown in Fig. 7. As two maxima move away, the interaction of them weakens. In Fig. 9, we show the calculated pulse shapes and spectra using the filter curve with $\omega_{\mathrm{m}}=7.0$. Comparing the results to Fig. 8, the spectrum here is less distorted and the intensity difference of two fronts is also smaller than those in Fig. 8. Numerical results match well with the experiments. We use the frequency deviation $\Delta \omega$ of spectral peak from the center of the spectral pedestal as a parameter to represent how much the pulse is distorted by the complicated filter. The calculated $\Delta \omega$ versus $\omega_{\mathrm{m}}$ is also plotted in Fig. 9. From the plot, it is clear that as the interaction of two maxima weakens, the pulse becomes less and less distorted. Numerical results confirm that the spectral filtering has strong influence on DSR pulses. A saddle shape spectral response, which arises from, e.g, interaction of two filters, can lead to the asymmetry of DSR pulses.

\section{CONCLUSiOnS}

Pulse energy is one of the most important performance we are chasing for ultrafast fiber lasers. The larger the achievable pulse energy is, the better fiber laser it is. People have discovered and developed different pulse shaping mechanisms in fiber lasers targeting larger pulse energy achievable. We have summarized various pulse shaping mechanisms from anomalous dispersion-nonlinearity balance for traditional soliton generation, dispersion-management for stretch-pulse generation, self-similar evolution for similariton generation, spectral filtering for dissipative soliton generation, to peak-power-clamping for DSR operation in fiber lasers. So-far the DSR operation in fiber lasers is the most potential way to obtain unlimited pulse energy. Details of asymmetric DSR pulses with tilt-top are explored. Two fronts of the DSR pulse show different intensity and the pulses present tilted top. Spectrally, the peak wavelength of the pulses does not accord with the center of the rectangular pedestal. The more the spectrum is distorted, the larger intensity disparity two fronts exhibit. Numerical simulations match well with experiments, revealing that the spectral filtering has strong influence on the DSR pulses. The experimental and theoretical demonstration of tilt-top DSR pulses gives important new insight into the spectral filtering and the DSR generation.

\section{ACKNOWLEDGMENT}

L. Zhao and D. Li contributed equally to this work. D. Li would like to thank the China Scholarship Council for sponsorship. The work is supported in part by the National Natural Science Foundation of China under Grant 11674133, 11711530208, 61575089, and 61405079 , in part by the Royal Society [grant number IE161214], in part by the Jiangsu Province Science Foundation under Grant BK20140231, in part by the Key Laboratory of Optoelectronic Devices and Systems of Ministry of Education and Guangdong Province.

\section{REFERENCES}

[1] A. Hasegawa and F. Tappert, "Transmission of stationary nonlinear optical pulses in dispersive dielectric fibres. I. Anomalous dispersion," Appl. Phys. Lett., vol. 23, no. 1, pp. 142-144, Aug. 1973.

[2] L. F. Mollenauer, R. H. Stolen and J. P. Gordon, "Experimental observation of picosecond pulse narrowing and solitons in optical fibers," Phys. Rev. Lett., vol. 45, no. 13, pp. 1095-1098, Sep. 1980.

[3] J. D. Kafka, D. W. Hall and T. Baer, "Mode-locked erbium-doped fiber laser with soliton pulse shaping," Opt. Lett., vol. 14, no. 22, pp. 1269-1271, Nov. 1989.

[4] M. E. Fermann, M. Hofer, F. Haberl and S. P. Craig-Ryan, "Femtosecond fibre laser," Electron. Lett., vol. 26, no. 20, pp. 1737-1738, Sep. 1990.

[5] D. J. Richardson, R. I. Laming, D. N. Payne, M. W. Phillips and V. J. Matsas, "320 fs soliton generation with passively mode-locked erbium fibre laser," Electron. Lett., vol. 27, no. 9, pp. 730-732, Apr. 1991.

[6] K. Tamura, H. A. Haus, and E. P. Ippen, "Self-starting additive pulse mode-locked erbium fibre ring laser," Electron. Lett., vol. 28, no. 24, pp. 2226-2228, Nov. 1992.

[7] V. E. Zakharov and A. B. Shabat, "Exact theory of two dimensional self-focusing and one-dimensional self-modulation of waves in nonlinear media," Sov. Phys. JETP, vol. 34, no. 1, pp. 62-69, Jan. 1972.

[8] W. H. Renninger, A. Chong and F. W. Wise, "Area theorem and energy quantization for dissipative optical solitons," J. Opt. Soc. Am. B, vol. 27, no. 10, pp. 1978-1982, Oct. 2010.

[9] K. Tamura, E. P. Ippen, H. A. Haus and L. E. Nelson, "77-fs pulse generation from a stretched-pulse mode-locked all-fiber ring laser," Opt. Lett., vol. 18, no. 13, pp. 1080-1082, Jul. 1993.

[10] L. M. Zhao, D. Y. Tang, H. Y. Tam and C. Lu, "Pulse breaking recovery in fiber lasers," Opt. Express, vol. 16, no. 16, pp. 12102-12107, Aug. 2008.

[11] F. Ö. Ilday, F. W. Wise and T. Sosnowski, "High-energy femtosecond stretched-pulse fiber laser with a nonlinear optical loop mirror," Opt. Lett., vol. 27, no. 17, pp. 1531-1533, Sep. 2002.

[12] F. Ö. Ilday and F. W. Wise, "Nonlinearity management: a route to high-energy soliton fiber lasers," J. Opt. Soc. Am. B, vol. 19, no. 3, pp. 470-476, Mar. 2002.

[13] D. Anderson, M. Desaix, M. Karlsson, M. Lisak and M. L. Quiroga-Teixeiro, "Wave-breaking-free pulses in nonlinear-optical fibers," J. Opt. Soc. Am. B, vol. 10, no. 7, pp. 1185-1190, Jul. 1993.

[14] M. E. Fermann, V. I. Kruglov, B. C. Thomsen, J. M. Dudley and J. D. Harvey, "Self-similar propagation and amplification of parabolic pulses in optical fibers," Phys. Rev. Lett., vol. 84, no. 26, pp. 6010-6013, Jun. 2000.

[15] V. I. Kruglov, A. C. Peacock, J. M. Dudley and J. D. Harvey, "Self-similar propagation of high-power parabolic pulses in optical fiber amplifiers," Opt. Lett., vol. 25, no. 24, pp. 1753-1755, Dec. 2000.

[16] V. I. Kruglov, A. C. Peacock, J. D. Harvey and J. M. Dudley, "Self-similar propagation of parabolic pulses in normal-dispersion fiber amplifiers," J. Opt. Soc. Am. B, vol. 19, no. 3, pp. 461-469, Mar. 2002.

[17] A. C. Peacock, R. J. Kruhlak, J. D. Harvey and J. M. Dudley, "Solitary pulse propagation in high gain optical fiber amplifiers with normal group velocity dispersion," Opt. Comm., vol. 206, pp. 171-177, May. 2002.

[18] F. Ö. Ilday, J. Buckley, W. Clark and F. Wise, "Self-similar evolution of parabolic pulses in a laser," Phys. Rev. Lett., vol. 92, no. 21, pp. 213902, May. 2004.

[19] F. W. Wise, A. Chong and W. H. Renninger, "High-energy femtosecond fiber lasers based on pulse propagation at normal dispersion," Laser \& Photonics Rev., vol. 2, no. 1-2, pp. 58-73, Apr. 2008. 
[20] J. Buckley, F. W. Wise, F. Ö. Ilday and T. Sosnowski, "Femtosecond fiber lasers with pulse energies above $10 \mathrm{~nJ}$," Opt. Lett., vol. 30, no. 14, pp. 1888-1890, Jul. 2005.

[21] A. Hasegawa and F. Tappert, F. "Transmission of stationary nonlinear optical pulses in dispersive dielectric fibres. II. Normal dispersion," Appl. Phys. Lett., vol. 23, no. 3, pp. 171-173, Aug. 1973.

[22] P. A. Bélanger, L. Gagnon and C. Paré, "Solitary pulses in an amplified nonlinear dispersive medium," Opt. Lett., vol. 14, no. 17, pp. 943-945, Sep. 1989.

[23] G. P. Agrawal, Nonlinear Fiber Optics, 3rd ed. Academic Press, 2001, pp. 63-134.

[24] L. M. Zhao, D. Y. Tang and J. Wu, "Gain-guided soliton in a positive group dispersion fiber laser," Opt. Lett., vol. 31, no. 12, pp. 1788-1790, Jun. 2006.

[25] A. Chong, J. Buckley, W. Renninger and F. W. Wise, " All-normal-dispersion femtosecond fiber laser," Opt. Express, vol. 14, no. 21, pp. 10095-10100, Oct. 2006.

[26] W. Renninger, A. Chong and F.W.Wise, "Dissipative solitons in normal dispersion fiber lasers," Phys. Rev. A, vol. 77, no. 2, pp. 023814, Feb. 2008.

[27] L. M. Zhao, D. Y. Tang, T. H. Cheng and C. Lu, "Gain-guided solitons in dispersion-managed fiber lasers with large net cavity dispersion," Opt. Lett., vol. 31, no. 20, pp. 2957-2959, Oct. 2006.

[28] D. Y. Tang and L. M. Zhao, "Generation of 47-fs pulses directly from an erbium-doped fiber laser," Opt. Lett., vol. 32, no. 1, pp. 41-43, Jan. 2007.

[29] L. M. Zhao, D. Y. Tang, T. H. Cheng and C. Lu, "Ultrashort pulse generation in lasers by nonlinear pulse amplification and compression," Appl. Phys. Lett., vol. 90, no. 5, pp. 051102, Jan. 2007.

[30] L. M. Zhao, D. Y. Tang, J. Wu, X. Q. Fu and S. C. Wen, "Noise-like pulse in a gain-guided soliton fiber laser," Opt. Express, vol. 15, no. 5, pp. 2145-2150, Mar. 2007.

[31] L. M. Zhao, D. Y. Tang, T. H. Cheng, H. Y. Tam and C. Lu, "Generation of multiple gain-guided solitons in a fiber laser," Opt. Lett., vol. 32, no. 11, pp. 1581-1583, Jun. 2007.

[32] L. M. Zhao, D. Y. Tang, X. Wu, D. J. Lei and S. C. Wen, "Bound states of gain-guided solitons in a passively mode-locked fiber laser," Opt. Lett., vol. 32, no. 21, pp. 3191-3193, Nov. 2007.

[33] L. M. Zhao, D. Y. Tang, T. H. Cheng, H. Y. Tam and C. Lu, "Passive harmonic mode locking of gain-guided solitons in Erbium-doped fiber lasers," Chinese Sci. Bull., vol. 53, no. 5, pp. 676-680, Mar. 2008.

[34] L. M. Zhao, D. Y. Tang, X. Wu and H. Zhang, "Period-doubling of gain-guided solitons in fiber lasers of large net normal dispersion," Opt. Commun., vol. 281, no, 13, pp. 3557-3560, Jul. 2008.

[35] L. M. Zhao, D. Y. Tang, X. Wu, and H. Zhang, "Dissipative soliton trapping in fiber lasers of normal dispersion," Opt. Lett., vol. 35, no, 11, pp. 1902-1904, Jun. 2010.

[36] L. M. Zhao, D. Y. Tang, H. Zhang, T. H. Cheng, H. Y. Tam and C. Lu, "Dynamics of gain-guided solitons in an all-normal-dispersion fiber laser," Opt. Lett., vol. 32, no, 13, pp. 1806-1808, Jul. 2007.

[37] L. M. Zhao, D. Y. Tang, X. Wu, H. Zhang, C. Lu and H. Y. Tam, "Dynamics of gain-guided solitons in a dispersion-managed fiber laser with large normal cavity dispersion," Opt. Commun., vol. 281, no, 12, pp. 3324-3326, Jun. 2008.

[38] L. M. Zhao, C. Lu, H. Y. Tam, P. K. A. Wai and D. Y. Tang, "Gain dispersion for dissipative soliton generation in all-normal-dispersion fiber lasers," Appl. Optics, vol. 48, no. 27, pp. 5131-5137, Sep. 2009.

[39] Ge Yanqi, Luo Jiaolin, Zhang Shumin, Tang Dingyuan, Shen Deyuan and Zhao Luming, "Cavity-induced peak power clamping effect for gain-guided solitons in passively mode-locked fiber lasers," Infrared and Laser Eng., vol. 43, pp. 3533-3539, Nov. 2014.

[40] Ge Yanqi, Luo Jiaolin, Zhang Shumin, Tang Dingyuan, Shen Deyuan and Zhao Luming, "Transient process of dissipative soliton generation in normal dispersion fiber lasers," Chin. J. Lasers, vol. 40, no. 10, pp. 1005006, Oct. 2013.

[41] A. Chong, W. Renninger and F. W. Wise, "Properties of normal-dispersion femtosecond fiber lasers," J. Opt. Soc. Amer. B, vol. 25, no. 2, pp. 140-148, Feb. 2008.

[42] K. Kieu, W. H. Renninger, A. Chong and F. W. Wise, "Sub-100 fs pulses at watt-level powers from a dissipative-soliton fiber laser," Opt. Lett., vol. 34, no, 5, pp. 593-595, Mar. 2009.

[43] S. Lefrancois, K. Kieu, Y. Deng, J. D. Kafka, and F. W Wise, "Scaling of dissipative-soliton fiber lasers to megawatt peak powers by use of large area photonic-crystal fiber," Opt. Lett., vol. 35, no. 10, pp. 1569-1571, May. 2010.

[44] M. Baumgartl, B. Ortaç, C. Lecaplain, A. Hideur, J. Limpert and A. Tünnermann, "130 nJ 77 fs dissipative soliton fiber laser," Conference on Lasers and Electro-Optics 2010, OSA Technical Digest (CD) (Optical Society of America, 2010), paper CFK3. Available: https://www.osapublishing.org/abstract.cfm?uri=CLEO-2010-CFK3

[45] L. M. Zhao, D. Y. Tang, X. Wu and H. Zhang, "Dissipative soliton generation in an Yb-fiber laser with an invisible intra cavity bandpass filter," Opt. Lett., vol. 35, no. 16, pp. 2756-2758, Aug. 2010.

[46] L. M. Zhao, D. Y. Tang, H. Zhang, X. Wu, Qiaoliang Bao and Kian Ping Loh, "Dissipative soliton operation of an ytterbium-doped fiber laser mode locked with atomic multi-layer graphene," Opt. Lett., vol. 35, no. 21, pp. 3622-3624, Nov. 2010.

[47] J. L. Luo, Y. Q. Ge, D. Y. Tang, S. M. Zhang, D. Y. Shen and L. M. Zhao, "Mechanism of spectrum moving, narrowing, broadening, and wavelength switching of dissipative solitons in all-normal-dispersion Yb-fiber lasers," IEEE Photonics J., vol. 6, no. 1, pp. 1500608, Feb. 2014.

[48] W. H. Renninger, A. Chong and F. W. Wise, "Self-similar pulse evolution in an all-normal-dispersion laser," Phys. Rev. A, vol. 82, no. 2, pp. 021805, Aug. 2010.

[49] W. Chang, A. Ankiewicz, J. M. Soto-Crespo and N. Akhmediev, "Dissipative soliton resonances," vol. 78, no. 2, pp. 023830, Aug. 2008.

[50] W. Chang, J. M. Soto-Crespo, A. Ankiewicz, and N. Akhmediev, "Dissipative soliton resonances in the anomalous dispersion regime," Phys. Rev. A, vol. 79, no. 3, pp. 033840, Mar. 2009.

[51] D. Li, D. Tang, L. Zhao and D. Shen, "Mechanism of dissipative-soliton-resonance generation in passively mode-locked all-normal-dispersion fiber lasers," J. Lightwave Technol., vol. 33, no, 18, pp. 3781-3787, Sep. 2015.

[52] E. Ding, P. Grelu and J. N. Kutz, "Dissipative soliton resonance in a passively mode-locked fiber laser," Opt. Lett., vol. 36, no. 7, pp. 1146-1148, Apr. 2011.

[53] A. Komarov, F. Amrani, A. Dmitriev, K. Komarov and F. Sanchez, "Competition and coexistence of ultrashort pulses in passive mode-locked lasers under dissipative-soliton-resonance conditions," Phys. Rev. A, vol. 87, no. 2, pp. 02838, Feb. 2013.

[54] X. Wu, D. Y. Tang, H. Zhang and L. M. Zhao, "Dissipative soliton resonance in an all-normal-dispersionerbium-doped fiber laser," Opt. Express, vol. 17, no. 7, pp. 5580-5584, Mar, 2009.

[55] S. K. Wang, Q. Y. Ning, A. P. Luo, Z. B. Lin, Z. C. Luo and W. C. Xu, "Dissipative soliton resonance in a passively mode-locked figure-eight fiber laser," Opt. Express, vol. 21, no. 2, pp. 2402-2407, Jan. 2013.

[56] L. M. Zhao, A. C. Bartnik, Q. Q. Tai and F. W. Wise, "Generation of 8 $\mathrm{nJ}$ pulses from a dissipative-soliton fiber laser with a nonlinear optical loop mirror," Opt. Express, vol. 38, no. 11, pp. 1942-1944, Jun. 2013.

[57] L. Mei, G. Chen, L. Xu, X. Zhang, C. Gu, B. Sun and A. Wang, "Width and amplitude tunable square-wave pulse in dual-pump passivelymode-lockedfiberlaser," Opt. Lett., vol. 39, no. 11, pp. 3235-3237, Jun. 2014.

[58] D. J. Li, L. Li, J. Y. Zhou, L. M. Zhao, D. Y. Tang and D. Y. Shen, "Characterization and compression of dissipative-soliton-resonance pulses in fiber lasers," Sci. Rep., vol. 6, pp. 23631, Mar. 2016.

[59] Tuanjie Du, Zhengqian Luo, Runhua Yang, Yizhong Huang, Qiujun Ruan, Zhiping Cai and Huiying $\mathrm{Xu}$, "1.2-W average-power, $700-\mathrm{W}$ peak-power, 100-ps dissipative soliton resonance in a compact Er:Yb co-doped double-clad fiber laser," Opt. Lett., vol. 42, no. 3, pp. 462-465, Feb. 2017.

[60] Georges Semaan, Fatma Ben Braham, Jorel Fourmont, Mohamed Salhi, Faouzi Bahloul and François Sanchez, "10 $\mu \mathrm{J}$ dissipative soliton resonance square pulse in a dual amplifier figure-of-eight double-clad Er:Yb mode-locked fiber laser," Opt. Lett., vol. 41, no. 20, pp. 4767-4770, Oct. 2016.

[61] J. Zhao, D. Ouyang, Z. Zheng, M. Liu, X. Ren, C. Li, S. Ruan and W. $\mathrm{Xie}$, "100 W dissipative soliton resonances from a thulium-doped double-clad all-fiber-format MOPA system," Opt. Express, vol. 24, no. 11, pp. 12072-12081, May. 2016.

[62] I. Armas-Rivera, C. Cuadrado-Laborde, A. Carrascosa, E. A. Kuzin, G. Beltrán-Pérez, A. Díez and M. V. Andrés, "Dissipative soliton resonance in a full polarization-maintaining fiber ring laser at different values of dispersion," Opt. Express, vol. 24, no. 9, pp. 9966-9974, Apr. 2016. 
[63] C. Cuadrado-Laborde, I. Armas-Rivera, A. Carrascosa, E. A. Kuzin, G. Beltrán-Pérez, A. Díez and M. V. Andrés, "Instantaneous frequency measurement of dissipative soliton resonant light pulses," Opt. Lett., vol. 41, no. 24, pp. 5704-5707, Dec. 2016.

[64] J. M. Soto-Crespo and N. Akhmediev, "Composite solitons and two-pulse generation in passively mode-locked lasers modeled by the complex quintic Swift-Hohenberg equation," Phys. Rev. E, vol. 66, no. 6, pp. 066610, Dec. 2002. 